\title{
Receptive Fields for Generalized Map Pyramids: The Notion of Generalized Orbit
}

\author{
Carine Grasset-Simon, Guillaume Damiand, and Pascal Lienhardt \\ SIC, FRE-CNRS 2731 - Université de Poitiers, \\ bât. SP2MI, Bvd M. et P. Curie, \\ BP 30179, 86962 Futuroscope Chasseneuil Cedex - France \\ \{simon, damiand, lienhardt\}@sic.univ-poitiers.fr
}

\begin{abstract}
A pyramid of $n$-dimensional generalized maps is a hierarchical data structure. It can be used, for instance, in order to represent an irregular pyramid of $n$-dimensional images. A pyramid of generalized maps can be built by successively removing and/or contracting cells of any dimension. In this paper, we define generalized orbits, which extend the classical notion of receptive fields. Generalized orbits allow to establish the correspondence between a cell of a pyramid level and the set of cells of previous levels, the removal or contraction of which have led to the creation of this cell. In order to define generalized orbits, we extend, for generalized map pyramids, the notion of connecting walk defined by Brun and Kropatsch.
\end{abstract}

Keywords. Irregular pyramids, generalized maps, generalized map pyramids, connecting walks, generalized orbits.

\section{Introduction}

For image analysis, it can be useful for some applications to segment an image at different levels. According to the application, some informations appear more clearly at some levels. An image pyramid corresponds to several segmentation levels of an image; levels 0 corresponds to the original image, the following levels correspond to the successive segmentations of this image. Many works deal with 2D image regular pyramids (cf. e.g. [1]) or 2D image irregular pyramids (cf. e.g. $[2,3,4]$ ).

Most image processing algorithms need to extract informations from images, for instance the adjacency between regions of images (e.g. an algorithm of segmentation by region aggregation). Order is another interesting notion. For example, it can be useful to retrieve the order of the edges which compose the boundary of a $2 \mathrm{D}$ region, or in $3 \mathrm{D}$, to know the order of volumes and faces around an edge or a vertex.

Many definitions of irregular pyramids are based upon graphs [3, 5]. More recently, Brun and Kropatsch [6, 7, 8, 9] have studied 2D combinatorial map pyramids, since combinatorial maps allow to represent the whole topological in- 
formation of subdivisions of orientable surfaces without boundary (for instance the order information is generally not represented by graphs).

$3 \mathrm{D}$ and $4 \mathrm{D}$ images (time being the $4^{\text {th }}$ dimension) are now usual images. So, we want to extend the previous works for any dimension, by defining pyramids of generalized maps [10]. The $n$-dimensional generalized maps (or $n$-G-maps) represent the topology of $n$-dimensional quasi-manifolds [11, orientable or not, with or without boundary. We have chosen generalized maps since their definition is homogeneous for all dimensions. So, we can easily define generic operations and algorithms.

A pyramid of $n$-G-maps can be constructed in the following way. Given an $n$-G-map which represents for instance an image, each level of the pyramid is deduced from the previous level by applying simultaneously removals and/or contractions of cells of any dimension 1 .

It is essential for many applications, to establish the correspondence between a cell at a given level, and the set of cells of previous levels the removal or the contraction of which has led to the creation of this cell. In $2 \mathrm{D}$ for instance, it can be useful to associate a face with the corresponding region of a lower level. In particular, it allows to retrieve any information contained in a lower pyramid level. The notion of receptive field has been introduced in order to establish this correspondence between regions of different levels. First, this notion has been defined in the context of graph hierarchy 3 , in the following way: the receptive field of a cell of level $n$ is the set of all pixels of level 0 which "compose" this cell. More recently, Brun and Kropatsch define the notion of receptive field of a dart for $2 \mathrm{D}$ combinatorial map pyramid 12 . This notion is based on the notion of connecting walk between a surviving dart and its successor at a given level, which is the set of darts which separate these two darts at the previous level. The notion of reduction window generalizes that notion of connecting walks for any levels.

The main result presented in this paper is the definition of generalized orbits of $n$-G-map pyramids which makes it possible to associate any cell of any dimension of a given level with the set of corresponding cells of any lower level. This definition is based upon a generalization of the connecting walk notion, which is itself based upon the operation of "simultaneous removals and contractions of cells" presented in [13].

The notion of orbit is a classical one for combinatorial and generalized maps. It allows to define cells as set of darts, darts being the unique type of elements defining maps (c.f. section 2). We generalize the notion of receptive field for any orbit (i.e. any cell) and any levels by defining generalized orbit. With this notion, we retrieve for $n$-cells the union of $n$-cells at a lower level which have been "merged" in a unique $n$-cell in a upper level.

This paper is organized in the following way. We give in section 2 a brief recall about pyramids of $n$-dimensional generalized maps. The connecting walk

\footnotetext{
${ }^{1}$ Note that $2 \mathrm{D}$ combinatorial map pyramids as defined by Brun and Kropatsch are built in a particular way: each odd (resp. even) level is deduced from the previous one by contracting (resp. removing) edges.
} 
notion is defined in section 3, and generalized orbits are defined in section 4, At last, we conclude and give some perspectives in section 5 .

\section{Recall: Pyramids of $\boldsymbol{n}$-Dimensional Generalized Maps}

An $n$-dimensional generalized map is a set of abstract elements, called darts, together with applications defined on these darts (c.f. figure 1):

Definition 1 ( $\boldsymbol{n}$-G-map). Let $n \geq 0$. An n-dimensional generalized map $G$ (or $n$-G-map) is defined by $G=\left(D, \alpha_{0}, \ldots, \alpha_{n}\right)$ where:

1. $D$ is a finite set of darts;

2. $\forall i, 0 \leq i \leq n, \alpha_{i}$ is an involution 2 on $D$;

3. $\forall i, j, 0 \leq i<i+2 \leq j \leq n, \alpha_{i} \alpha_{j}$ is an involution.

The $n$-G-maps represent the topology of subdivided objects, more precisely the topology of quasi-manifolds (see [11]). Cells are implicitly represented as subset of darts:

Definition 2 ( $\boldsymbol{i}$-cell). Let $G$ be an $n-G$-map, $d$ be a dart and $i \in N=\{0, \ldots, n\}$. The i-cell incident to $d$ is the orbi

$$
<>_{N-\{i\}}(d)=<\alpha_{0}, \ldots, \alpha_{i-1}, \alpha_{i+1}, \ldots, \alpha_{n}>(d) .
$$

Figure 1 illustrates the notions of generalized map and $i$-cell. Intuitively, an $i$-cell is the set of all darts which can be reached starting from $d$, by using any combination of all involutions except $\alpha_{i}$. The set of $i$-cells is a partition of the set of darts $D$, for each $i$ between 0 and $n$. Two cells are disjoint when their intersection is empty, i.e. when no dart is shared by the cells. More precisions about $n$-G-maps are provided in [11] and [14.

In order to define $n$-G-map pyramids, Damiand and Lienhardt have defined the operation of "simultaneous removals and contractions of cells of any dimension" 13] which allows to contract and remove a set of cells of any dimension in a simultaneous way. The formal definition of this operation is:

\section{Definition 3 (Simultaneous Removal and Contraction of Cells of Any Dimension).}

Let $G=\left(D, \alpha_{0}, \ldots, \alpha_{n}\right)$ be an $n-G$-map, $R_{0}, \ldots, R_{n-1}$ be sets of 0 -cells, .. , $(n-1)$-cells to be removed and $C_{1}, \ldots, C_{n}$ be sets of 1 -cells, $\ldots, n$-cells to be contracted. Let $R=\cup_{i=0}^{n-1} R_{i}$ and $C=\cup_{i=1}^{n} C_{i}$. Two preconditions have to be satisfied: cells are disjoint (i.e. $\forall c, c^{\prime} \in C \cup R, c \cap c^{\prime}=\emptyset$ ), and "the degree of each cell is equal to 2", i.e.:

${ }^{2}$ An involution $f$ on a finite set $S$ is a one to one mapping from $S$ onto $S$ such that $f=f^{-1}$.

${ }^{3}$ Let $\left\{\Pi_{0}, \ldots, \Pi_{n}\right\}$ be a set of permutations on D. The orbit of an element $d$ relatively to this set of permutations is $<\Pi_{0}, \ldots, \Pi_{n}>(d)=\left\{\Phi(d), \Phi \in<\Pi_{0}, \ldots, \Pi_{n}>\right\}$, where $<\Pi_{0}, \ldots, \Pi_{n}>$ denotes the group of permutations generated by $\Pi_{0}, \ldots, \Pi_{n}$. 


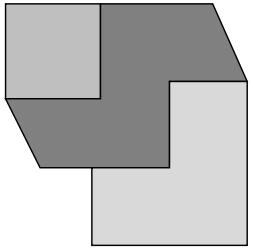

(a)

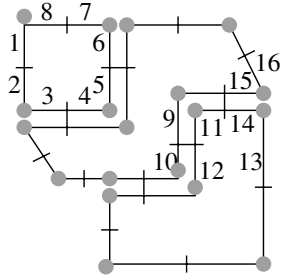

(b)

Fig. 1. (a) A subdivision of a surface. (b) The corresponding 2-G-map. Darts are represented by (numbered) black segments. Two darts in relation by $\alpha_{0}$ share a little vertical segment (ex. darts 1 and 2). Two darts in relation by $\alpha_{1}$ share a same point (ex. darts 2 and 3). Two distinct darts in relation by $\alpha_{2}$ are parallel and close to each other (ex. darts 9 and 11); otherwise, the dart is its own image by $\alpha_{2}$ (ex. dart $2)$. The vertex incident to dart 14 is $\left\langle\alpha_{1}, \alpha_{2}\right\rangle(14)=\{13,14,15,16\}$, the edge incident to dart 9 is $\left\langle\alpha_{0}, \alpha_{2}>(9)=\{9,10,11,12\}\right.$, and the face incident to dart 4 is $<\alpha_{0}, \alpha_{1}>(4)=\{1,2,3,4,5,6,7,8\}$

- $\forall i, 0 \leq i \leq n-2, \forall d \in R_{i}, d \alpha_{i+1} \alpha_{i+2}=d \alpha_{i+2} \alpha_{i+1}$

- $\forall i, 2 \leq i \leq n, \forall d \in C_{i}, d \alpha_{i-1} \alpha_{i-2}=d \alpha_{i-2} \alpha_{i-1}$

Let $S D_{i}=\left(R_{i} \cup C_{i}\right) \alpha_{i}-\left(R_{i} \cup C_{i}\right) \forall i, 0 \leq i \leq n$ (it is the set of surviving darts "neighbour" of removed and contracted cells). The resulting $n-G$-map is $G^{\prime}=\left(D^{\prime}, \alpha_{0}^{\prime}, \ldots, \alpha_{n}^{\prime}\right)$ defined by:

$-D^{\prime}=D-(C \cup R)$;

$-\forall i, 0 \leq i \leq n, \forall d \in D^{\prime}-S D_{i}, d \alpha_{i}^{\prime}=d \alpha_{i}$

$-\forall i, 0 \leq i \leq n, \forall d \in S D_{i}, d \alpha_{i}^{\prime}=d^{\prime}=d\left(\alpha_{i} \alpha_{k_{1}}\right) \ldots\left(\alpha_{i} \alpha_{k_{p}}\right) \alpha_{i}$, where $p$ is the smallest integer such that $d^{\prime} \in S D_{i}$, and $\forall j, 1 \leq j<p$, if $d_{j}=$ $d\left(\alpha_{i} \alpha_{k_{1}}\right) \ldots\left(\alpha_{i} \alpha_{k_{j-1}}\right) \alpha_{i} \in R_{i}$ then $k_{j}=i+1$ else $\left(d_{j} \in C_{i}\right) k_{j}=i-1$.

Figure 2 illustrate this operation.

A pyramid of $n$-dimensional generalized maps (or $n$-G-map pyramid) is a hierarchical data structure [10]. Each level is an n-G-map, deduced from the previous level by applying the general operation of removal and/or contraction of cells. The choice of the removed or contracted cells depends on the application (we assume here that this choice is the result of an external process). An $n$-G-map pyramid can be defined in the following way (see figure 3 a):

Definition 4 (n-G-map Pyramid). Let $n, m \geq 0$. An $m+1$ level pyramid $\mathcal{P}$ of $n$-dimensional generalized maps is defined by $\mathcal{P}=\left\{G^{k}\right\}_{0 \leq k \leq m}$ where:

1. for each $k, 0 \leq k \leq m, G^{k}=\left(D^{k}, \alpha_{0}^{k}, \ldots, \alpha_{n}^{k}\right)$ is an $n$-G-map;

2. for each level $k, 0<k \leq m$, 


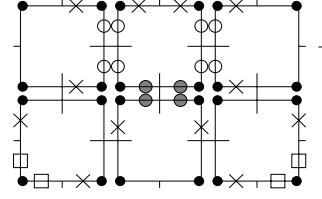

a

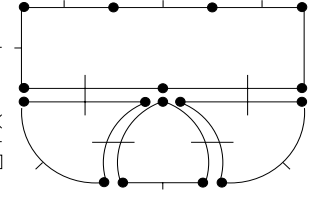

b

Fig. 2. An example of simultaneous removal and contraction of cells of different dimensions. (a) A 2-G-map where the darts 0-removed, 1-removed and 1-contracted are respectively marked by empty squares, circles and gray disks. The surviving "neighbour" darts are marked by crosses. (b) The 2-G-map obtained by application of the operation

- $R^{k-1}=\bigcup_{i=0}^{n-1} R_{i}^{k-1}$ (resp. $\left.C^{k-1}=\bigcup_{i=1}^{n} C_{i}^{k-1}\right)$ is a set of cells of $G^{k-1}$. These cells are disjoint, and their degrees equals to 2.

- $G^{k}$ is deduced from $G^{k-1}$ by simultaneously removing $R^{k-1}$ and contracting $C^{k-1}$.

In the following, $\mathcal{P}$ denotes an $n$-G-map pyramid composed of $m+1$ levels numbered from 0 to $m$. Level $k$ refers to $n$-G-map $G^{k}$. A dart keeps its name when it is not suppressed (for instance dart 1 of level 0 and 1 in figure $3-b$ ). So, $D^{k+1} \subseteq D^{k}$ for any $k, 0 \leq k<m$ : each dart appears for the first time in the first pyramid level; if a dart belongs to the $k^{\text {th }}$ pyramid level, it does not belong to a cell which is removed or contracted in a precedent level. Lev $v_{d}$ denotes the last level in which dart $d$ exists. At last, note that for a given dimension $i$ and a given level $k$, each dart $d$ in $D^{k}$ is either a dart which belongs to a removed $i$-cell $\left(d \in R_{i}^{k}\right)$ or to a contracted $i$-cell $\left(d \in C_{i}^{k}\right)$, or a dart which is a "neighbour" of a contracted or removed $i$-cell $\left(d \in S D_{i}^{k}\right)$, or an other dart $\left(d \notin\left(R_{i}^{k} \cup C_{i}^{k} \cup S D_{i}^{k}\right)\right)$.

\section{Connecting Walks}

The notion of connecting walk has been defined by Brun and Kropatsch for combinatorial map pyramids. More precisely, a connecting walk is the set of darts at a given level which separates a surviving dart and its successor in the next level. We extend this notion for $n$-G-map pyramids, for any two levels, and any dart of the pyramid. In the standard case (for surviving darts), the definition of connecting walk corresponds to that of Brun and Kropatsch. For all other darts of the pyramid (non surviving darts), this definition is extended in order to be able to define generalized orbits. A connecting walk is a sequence of darts in a lower level that separates two darts of a upper level. Intuitively, a connecting walk at a given level is obtained by concatenating connecting walks of the previous level concerned by removals or contractions. $C h_{(i, a, b)}(d)$ denotes the connecting walk between levels $a$ and $b(a \leq b)$, for any dart $d \in D^{a}$ and dimension $i$.

First, assume $d \in D^{b}$ (a surviving dart). $C h_{(i, a, b)}(d)$ is the sequence of darts at level $a$ separating dart $d$ and its neighbour $d \alpha_{i}^{b}$. 
When $b=a+1$, we get a definition near to that of Brun and Kropatsch. In fact, when $b=a+1, C h_{(i, a, a+1)}(d)$ is then the sequence of darts removed and contracted between levels $a$ and $a+1$ linking $d$ and $d \alpha_{i}^{a+1}$. These darts are traversed when we define $d \alpha_{i}^{a+1}$ (cf. definition 3 of simultaneous removal and contraction).

When $b>a+1, C h_{(i, a, b)}(d)$ is the sequence of darts removed and contracted between levels $a$ and $b$ linking $d$ and $d \alpha_{i}^{b}$. Using the definition of removals and contractions of cells, we can iterate the previous process for each level. So, we can express $C h_{(i, a, b)}(d)$ as a concatenation of walks examined between levels $a$ and $b-1$. We obtain a recursive definition of $C h_{(i, a, b)}(d)$.

In the particular case where there is no dart between $d$ and its neighbour for $\alpha_{i}^{b}$ at level $b-1$, that is to say where $d$ is not the neighbour for $\alpha_{i}^{b-1}$ of a contracted or removed dart, then $C h_{(i, a, b)}(d)$ is equal to $C h_{(i, a, b-1)}(d)$.

We can observe that $C h_{(i, a, a)}(d)$ is composed of only one dart: $d \alpha_{i}^{a}$, since no darts at level $a$ separates $d$ and $d \alpha_{i}^{a}$.

Second, assume $d \notin D^{b}$ (a non surviving dart).

We have to extend the notion of connecting walk in order to define the notion of generalized orbit. When $d \in D^{b-1}$, the removal or contraction of the cell containing $d$ is directly concerned in the construction of level $b$, and has direct consequences in the definition of new orbits of this level. This is not the case when $d \notin D^{b-1}$. For these reasons, if $d \notin D^{b-1}, C h_{(i, a, b)}(d)$ is the empty sequence, and if $d \in D^{b-1}, C h_{(i, a, b)}(d)$ corresponds to the sequence of darts traversed from $d$ and applying the same rules than for the definition of removal and contraction. There are here two conditions to stop: when the last dart $d^{\prime}$ belong to $D^{b}$, or when the last dart is $d$ (In the first case, $C h_{(i, a, b)}(d)$ corresponds to a subsequence or to the inverse of a subsequence of $C h_{(i, a, b)}\left(d^{\prime}\right)$. In the second case, $C h_{(i, a, b)}(d)$ is a cycle).

See figure 3 for examples of these different cases of connecting walks. The notion of connecting walk is formally defined in the following way:

Definition 5 (Connecting Walk). Let $i \in N, a$ and $b$ be such that $0 \leq a \leq$ $b \leq m$.

For each dart $d \in D^{a}, C h_{(i, a, b)}(d)$ is defined by:

if $b=a: C h_{(i, a, b)}(d)=\left(d \alpha_{i}^{a}\right)$,

else if $b>l e v_{d}+1: C h_{(i, a, b)}(d)=()$,

else if $d \notin\left(S D_{i}^{b-1} \cup R_{i}^{b-1} \cup C_{i}^{b-1}\right): C h_{(i, a, b)}(d)=C h_{(i, a, b-1)}(d)$,

else: $C h_{(i, a, b)}(d)=C=\left(C h_{\left(k_{1}, a, b-1\right)}\left(d_{1}\right), \ldots, C h_{\left(k_{p}, a, b-1\right)}\left(d_{p}\right)\right)$,

where: $d_{1}=d$,

$\forall u, 1 \leq u<p, d_{u+1}$ is the last dart of $C h_{\left(k_{u}, a, b-1\right)}\left(d_{u}\right)$,

$\forall u, 1 \leq u \leq p, k_{u}=\left\{\begin{array}{l}i \text { if } u \text { is odd } \\ i+1 \text { if } u \text { is even and } d_{u} \in R_{i}^{b-1} \\ i-1 \text { if } u \text { is even and } d_{u} \in C_{i}^{b-1},\end{array}\right.$

and $p$ is the smallest integer such that the last dart of $C$ is equal to $d$, or is a surviving dart. 


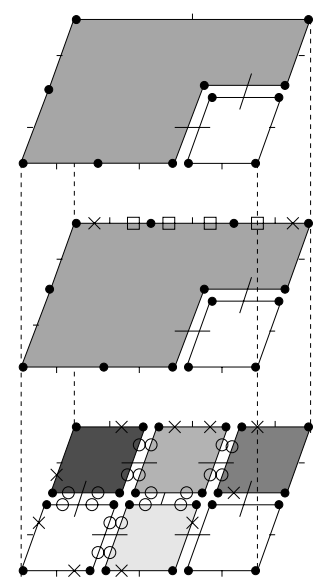

a
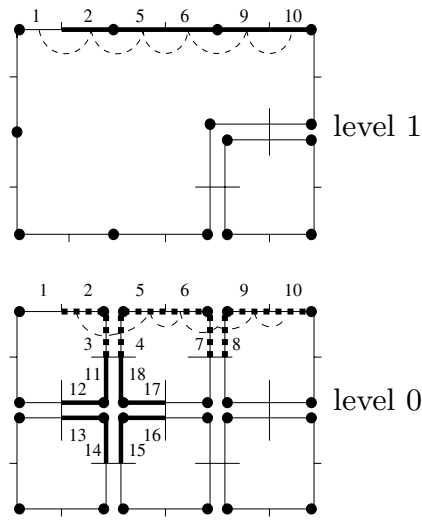

b

Fig. 3. (a) Example of 2-G-map pyramid composed of three levels. The darts 0-removed (resp. 1-removed) are marked by empty squares (resp. circles). (b) Two levels of the pyramid. The darts of connecting walk $C h_{(0,1,2)}(1)$ are drawn thick in level 1 . These darts are between darts 1 and 10 , where $10=1 \alpha_{0}^{2}: C h_{(0,1,2)}(1)=(2,5,6,9,10)=$ $\left(1 \alpha_{0}^{1}, 1 \alpha_{0}^{1} \alpha_{1}^{1}, 1 \alpha_{0}^{1} \alpha_{1}^{1} \alpha_{0}^{1}, 1 \alpha_{0}^{1} \alpha_{1}^{1} \alpha_{0}^{1} \alpha_{1}^{1}, 1 \alpha_{0}^{1} \alpha_{1}^{1} \alpha_{0}^{1} \alpha_{1}^{1} \alpha_{0}^{1}\right) . E c h_{(0,1,2)}^{\circ}(1)=\{2,5,6,9\}$, and $\operatorname{Ech}_{(0,1,2)}(1)=\{1,2,5,6,9,10\}$. The darts of connecting walk $C h_{(0,0,2)}(1)$ are drawn dotted in level 0 , and $C h_{(0,0,2)}(1)=(2,3,4,5,6,7,8,9,10) . C h_{(0,0,2)}(1)$ is the concatenation of $C h_{(0,0,1)}(1), C h_{(1,0,1)}(2), C h_{(0,0,1)}(5), C h_{(1,0,1)}(6)$ and $C h_{(0,0,1)}(9)$. Note that $C h_{(0,0,1)}(5)=C h_{(0,0,0)}(5)=(6)$. Darts 5 and 11 are non surviving darts at level 2: $C h_{(0,0,2)}(5)=(6,7,8,9,10)$ is a subsequence of $C h_{(0,0,2)}(1)$, and $C h_{(1,0,1)}(11)=$ $(12,13,14,15,16,17,18,11)$ is a cycle

From this definition, we deduce in a straightforward way an algorithm which computes a connecting walk with a cost $\Theta(n), n$ being the number of darts of the connecting walk.

Given a connecting walk $C h_{(i, a, b)}(d)$, we deduce two sets of darts: open connecting set $\operatorname{Ech}_{(i, a, b)}^{\circ}(d)$ and closed connecting set $\operatorname{Ech}_{(i, a, b)}(d)$ (c.f. figure 3-b). Intuitively, the first set corresponds to the interior of the connecting walk and the second set corresponds to the whole connecting walk, extremities included. The notion of generalized orbits is based upon these sets.

Definition 6 (Open and Closed Connecting Sets). Let $i \in N$, a and $b$ be such that $0 \leq a \leq b \leq m$.

For each dart $d \in D^{a}$ :

- $\operatorname{Ech}_{(i, a, b)}^{\circ}(d)$ is the set of darts of the connecting walk $C h_{(i, a, b)}(d)$, the last dart excepted;

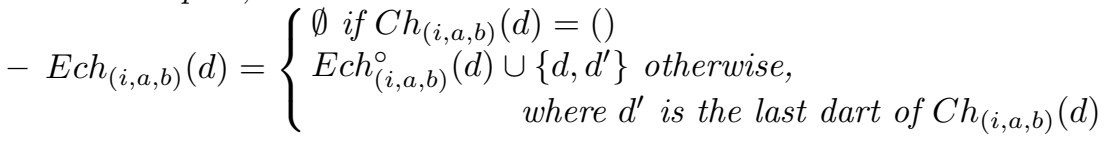




\section{Generalized Orbits}

A possible use of $n$-G-map pyramid is the representation of an image segmented at several levels. For instance, an $n$-G-map pyramid can be used for representing an $n \mathrm{D}$ image in gray level which is segmented using a simple gray level distance as homogeneous criterion. Level 0 of the pyramid represents the initial image. Each xel is represented by an $n$-cell associated with a gray level. At the following level, neighbour regions which satisfy the homogeneity criterion are merged into a unique region. Merging is achieved by removing $(n-1)$-cells which separate them (note that other operations are possible in order to simplify the boundary between two adjacent regions).

We can compute the gray level of an $n$-cell from the gray levels of all the $n$-cells at level 0 which correspond to this cell. For that we need to compute the set of $n$-cells at level 0 which correspond to a given $n$-cell.

More generally, let $a$ and $b$ be any two levels of an $n$-G-map pyramid $\mathcal{P}$ such that $0 \leq a \leq b \leq m$. Let $K \subseteq\{0, \ldots, n\}$ and let $O=<>_{(K)^{b}}(d)$ be an orbit of $G^{b}$, the $n$-G-map of the level $b$ of $\mathcal{P}$. The set of darts corresponding to $O$ at level $a$ is called generalized orbit. Informally, a generalized orbit is the set of orbits at level $a$ which are "merged" into the orbit at level $b$.

A generalized orbit can be computed in a sequential way (see figure 4). Let $G O$ be the generalized orbit associated to $O$. $G O$ is initialized by $G O_{0}=\{d\}$. Then we repeat the two following phases: first we add the darts of the connecting walks of the darts which belong to $G O$ (i.e. we define $G O_{2 p+1}$ as the union of $G O_{2 p}$ and the set of all darts of closed connecting set $E_{c h} h_{(i, a, b)}\left(d^{\prime}\right)$ for all dimension $i \in K$ and all darts $d^{\prime}$ of $G O_{2 p}$ ); second, we add all darts of the orbits $<>_{K}$ of darts belonging to $G O$ (i.e. we define $G O_{2 p+2}$ as the union of all orbits $\left\langle>_{(K)^{a}}\left(d^{\prime}\right)\right.$ for all darts $d^{\prime}$ of $\left.G O_{2 p+1}\right)$. Since an $n$-G-map contains a finite number of darts, we can show that $q \geq 0$ exists, such that no dart is added to $G O_{q(K, a, b)}(d)$ by repeating the process. Generalized orbit $G O$ is defined as $G O_{q(K, a, b)}(d)$.

Definition 7 (The Series $\left.\left(\boldsymbol{G} \boldsymbol{O}_{\boldsymbol{p}(\boldsymbol{K}, \boldsymbol{a}, \boldsymbol{b})}(\boldsymbol{d})\right)_{\mathbf{0} \leq \boldsymbol{p} \leq \boldsymbol{q}}\right)$. Let $K \subseteq N$, let $a$ and $b$ be such that $0 \leq a \leq b \leq m$, and let $d \in D^{b}$.

We define the series $\left(G O_{p(K, a, b)}(d)\right)_{0 \leq p \leq q}$ in the following way:

$-G O_{0(K, a, b)}(d)=\{d\}$

$-\forall n>0$ :

$$
\begin{aligned}
& G O_{2 p-1(K, a, b)}(d)=\bigcup_{i \in K} \bigcup_{d^{\prime} \in G O_{2 p-2(K, a, b)}(d)} \operatorname{Ech}_{\left(i, a, \min \left\{b, l e v_{d^{\prime}}+1\right\}\right)}\left(d^{\prime}\right) ; \\
& G O_{2 p(K, a, b)}(d)=<>(K)^{a} \\
& \left(G O_{2 p-1(K, a, b)}(d)\right) .
\end{aligned}
$$

Note that the even elements of this series are unions of orbits $<>_{K}$ (for instance, we can see in figure 5 that a generalized face is a set of faces). As we have said before, a property of this series is that it is convergent in a finite number of iterations. Indeed, it is increasing, bounded by $D^{a}$ and so stationary. We can thus define a generalized orbit as the limit of such a series. 


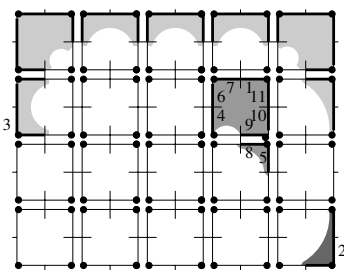

a

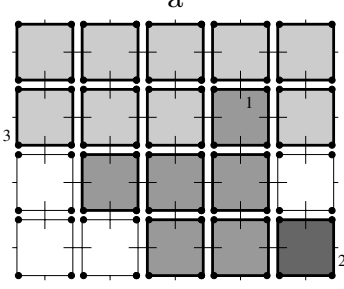

d

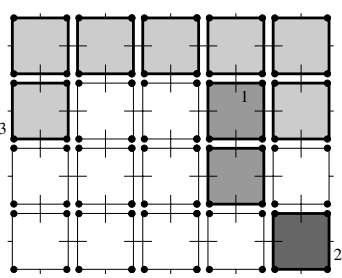

$\mathrm{b}$

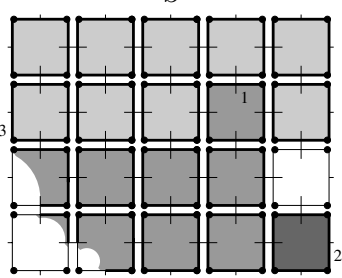

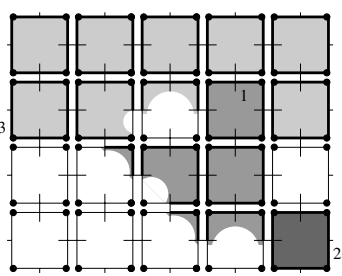

$\mathrm{c}$

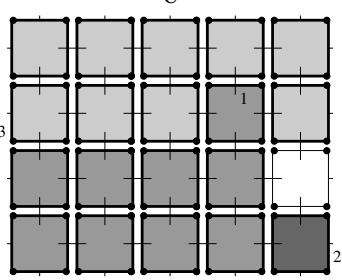

$\mathrm{f}$

Fig. 4. Computing the generalized orbit $<>_{K}=<>_{\{0,1\}}$ (the face orbit) for darts 1,2 and 3 in the pyramid of the figure [5 (a) $G O_{1(K, 0,5)}$ for darts 1, 2 and 3, are represented in different gray levels. (b) to (f) $G O_{j(K, 0,5)}$, for darts 1,2 and 3 , from $j=2$ to $j=6$, are represented in different gray levels. Note that $G O_{j(K, 0,5)}=G O_{6(K, 0,5)}$ for $j>6$. For example, when we compute $G O_{1(K, 0,5)}(1)$, we add the darts of $C h_{(0,0,5)}(1)=(7,6,4)$ and $C h_{(1,0,5)}(1)=(11,10,9,8,5)$

Definition 8 (Generalized Orbit). Let $K \subseteq N$, let $a$ and $b$ be such that $0 \leq a \leq b \leq m$, and let $d \in D^{b}$. The generalized orbit $G O_{(K, a, b)}(d)$ is defined as the limit of the series $\left(G O_{p(K, a, b)}(d)\right)_{0 \leq p \leq q}$.

From definitions 7 and 8 , we can deduce directly a first algorithm which computes a generalized orbit with a cost $\Theta\left(k n^{2}\right)$, where $n$ is the number of darts of this generalized orbit, and $k=\operatorname{card}(K)$.

The problem of this algorithm is to consider all darts at each step, whereas it is not useful. In order to optimize this algorithm, we can remark that:

1. it is necessary to consider each dart of the orbit for all involutions of this orbit. So the lower bound is $\Theta(k n)$.

2. when we have added the darts of $C h_{(i, a, b)}(d)$, it is not useful to consider the connecting walk for $\alpha_{i}$, of a dart of the interior of $C h_{(i, a, b)}(d)$ since it is included in $C h_{(i, a, b)}(d)$. Moreover a dart will not be considered another time for $\alpha_{i}$ since the intersection of two different connecting walks is empty.

So, we can propose an optimized algorithm that computes a generalized orbit with a cost $\Theta(k n)$. 


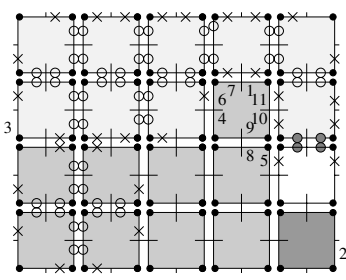

a

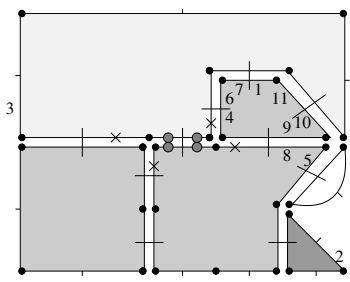

d

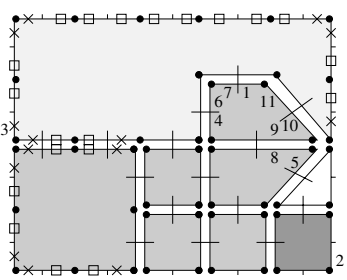

b

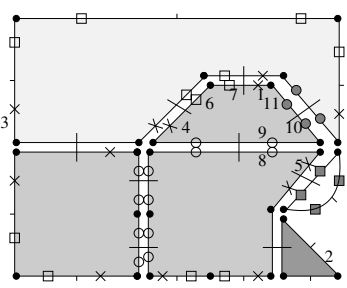

e

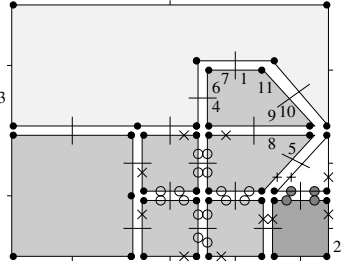

C

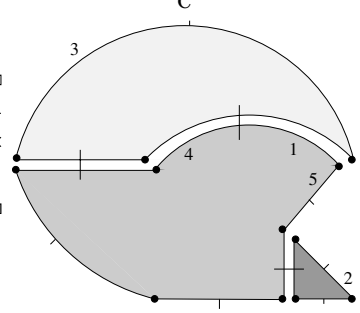

f

Fig. 5. A pyramid. a (resp. b, c, d, e, et f) represents level 0 (resp. 1, 2, 3, 4 et 5). Darts 0-removed (resp. 1-removed, 1-contracted and 2-contracted) are marked with empty squares (resp. circles, gray disks and gray squares). The faces of level 5 and the corresponding generalized faces in lower levels are represented in different gray level

Among the properties of generalized orbits we have:

- $\forall d \in D^{a}, G O_{(K, a, a)}(d)=<>_{(K) a}(d)$. Generalized orbits are an extension of orbits and we retrieve them when we consider generalized orbits at only one level;

- $G O_{(K, a, b)}(d)$ is a union of orbits $<>_{K}$ at level $a$ (by definition).

$n$-cells satisfy some additional properties. We can easily show that the $n$-cells of a level are necessarily the result of the "merging" of $n$-cells of the previous level by applying $(n-1)$-removals. All the other operations only modify the boundary of existing $n$-cells.

So the generalized orbit associated to an $n$-cell is the union of all $n$-cells of level $a$ which have been "merged" to construct it. We can note that even when $(n-1)$-removals have led to a disconnection (see figure 6), we retrieve for the generalized orbit associated to an $n$-cell, the set of $n$-cells at level $a$ which compose it. With this property, we can deduce that two generalized orbits associated with $n$-cells and to the same levels are either equal or disjoint.

These last properties are not necessarily true for other $i$-cells $(i \neq n)$. But, in general, the interesting cells that contain information are $n$-cells and not the others. In order to get such properties for the other cells, it could be necessary to define other sets of darts and perhaps other generalized orbits. 


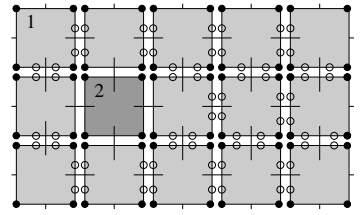

level 0

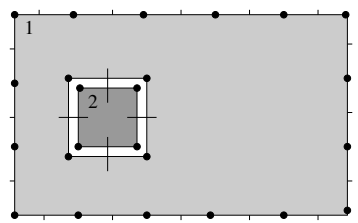

level 1

Fig. 6. A 2-G-map pyramid composed of two levels. 1-removed darts are marked by circles. The general orbits associated with faces for darts 1 and 2 are represented in two gray levels. Note in the second level that the removal of edges has led to a disconnection

\section{Conclusion and Perspectives}

We have defined the notion of generalized orbit which extends that of receptive field. This notion, defined for graph pyramids and combinatorial maps pyramids, establishes a correspondence between a region at a given level and the corresponding set of regions at a lower level. The notion of generalized orbit is defined for $n$-G-map pyramids, which can be used in order to represent pyramids of $n$-dimensional images. Moreover, this notion is defined for any cells of any dimension, and between any two levels. The definition of this notion is based upon a generalization of the connecting walk notion, initially defined by Brun and Kropatsch.

Some properties of generalized orbits have been established. Among them, two concern only $n$-cells. In order to define similar properties for other cells, we are going to define other connecting walks and generalized orbits, and compare them for different cells of different dimensions.

Moreover we are conceiving operations for handling $n$-G-map pyramids. More precisely, we want to be able to modify a level of a pyramid, and to automatically compute the modifications of the upper and lower levels. Last, given a generalized orbit and an operation which modifies the pyramid, we want to optimize the computation of the modified generalized orbit, i.e. to directly deduce it without re-computing it from scratch.

\section{Acknowledgements}

The authors wish to thank Luc Brun and Walter Kropatsch for their encouragements and help.

\section{References}

1. Burt, P., Hong, T., Rosenfeld, A.: Segmentation and estimation of image region properties through cooperative hierarchical computation. IEEE Transactions on Systems, Man and Cybernetics 11 (1981) 802-809. 
2. Meer, P.: Stochastic image pyramids. Computer Vision, Graphics and Image Processing 45 (1989) 269-294.

3. Montanvert, A., Meer, P., Rosenfeld, A.: Hierarchical image analysis using irregular tessellations. IEEE Transactions on Pattern Analysis and Machine Intelligence 13 (1991) 307-316.

4. Jolion, J., Montanvert, A.: The adaptive pyramid: A framework for $2 \mathrm{~d}$ image analysis. Computer Vision, Graphics and Image Processing 55 (1992) 339-348.

5. Kropatsch, W.: Building irregular pyramids by dual-graph contraction. Vision, Image and Signal Processing 142 (1995) 366-374.

6. Brun, L., Kropatsch, W.: Irregular pyramids with combinatorial maps. In Amin, A., Ferri, F.J., Pudil, P., I nesta, F.J., eds.: Advances in Pattern Recognition, Joint IAPR International Workshops SSPR'2000 and SPR'2000. Volume 1451 of Lecture Notes in Computer Science., Alicante, Spain, Springer, Berlin Heidelberg, New York (2000) 256-265.

7. Brun, L., Kropatsch, W.: Introduction to combinatorial pyramids. In G. Bertrand, A. Imiya, R.K., ed.: Digital and Image Geometry. Volume 2243 of LNCS. Springer Verlag (2001) 108-127.

8. Brun, L., Kropatsch, W.: Combinatorial pyramids. In Suvisoft, ed.: IEEE International conference on Image Processing (ICIP). Volume II., Barcelona (2003) $33-37$.

9. Brun, L., Kropatsch, W.: Contraction kernels and combinatorial maps. Pattern Recognition Letters 24 (2003) 1051-1057.

10. Grasset-Simon, C., Damiand, G., Lienhardt, P.: Pyramids of n-dimensional generalized map. Technical Report 2, SIC, Université de Poitiers (2004)

11. Lienhardt, P.: N-dimensional generalized combinatorial maps and cellular quasimanifolds. In: International Journal of Computational Geometry and Applications. (1994) 275-324.

12. Brun, L., Kropatsch, W.: Receptive fields within the combinatorial pyramid framework. In: Discrete Geometry for Computer Imagery. Number 2301 in LNCS, Bordeaux, France (2002) 92-101.

13. Damiand, G., Lienhardt, P.: Removal and contraction for n-dimensional generalized maps. In: Discrete Geometry for Computer Imagery. Number 2886 in Lecture Notes in Computer Science, Naples, Italy (2003) 408-419.

14. Lienhardt, P.: Subdivisions of n-dimensional spaces and n-dimensional generalized maps. In: Proceedings of the fifth annual Symposium on Computational Geometry, Saarbruchen, West Germany (1989) 228-236. 